\title{
NOTE ON STIRLING POLYNOMIALS
}

\author{
JUNESANG CHOI*
}

\begin{abstract}
A large number of sequences of polynomials and numbers have arisen in mathematics. Some of them, for example, Bernoulli polynomials and numbers, have been investigated deeply and widely. Here we aim at presenting certain interesting and (potentially) useful identities involving mainly in the second-order Eulerian numbers and Stirling polynomials, which seem to have not been given so much attention.
\end{abstract}

\section{Introduction and preliminaries}

Some sequences of polynomials arise often in mathematics that we give them special names which are mainly named after persons who invented, for example, Bernoulli polynomials, Euler polynomials, Genocchi polynomials, Hermite polynomials, Laguerre polynomials, Jacobi polynomials and so on (see, e.g., [8] and [10, Section 1.7]). Likewise, certain sequences of numbers appear so often in mathematics that we recognize them immediately and give them special names, for example, square numbers, triangular numbers, prime numbers, Bernoulli numbers, Euler numbers, Eulerian numbers, Stirling numbers, Fibonacci numbers, and so on (see, e.g., the references given here). We begin by recalling Bernoulli polynomials and numbers. The Bernoulli polynomials $B_{n}(x)$ are defined by the generating function:

$$
\frac{z e^{x z}}{e^{z}-1}=\sum_{n=0}^{\infty} B_{n}(x) \frac{z^{n}}{n !} \quad(|z|<2 \pi) .
$$

Received May 01, 2013; Accepted July 08, 2013.

2010 Mathematics Subject Classification: Primary 11B68, 11B73, 33B15; Secondary 33C05, 33C45.

Key words and phrases: Stirling numbers of the first and second kinds, Eulerian numbers, second-order Eulerian numbers, harmonic numbers, generalized harmonic numbers, gamma function, Hurwitz (or generalized) zeta function, Psi (or Digamma) function, Polygamma functions, Pochhammer symbol, Stirling polynomials. 
The numbers $B_{n}:=B_{n}(0)$ are called the Bernoulli numbers generated by

$$
\frac{z}{e^{z}-1}=\sum_{n=0}^{\infty} B_{n} \frac{z^{n}}{n !} \quad(|z|<2 \pi) .
$$

It easily follows from (1.1) and (1.2) that

$$
B_{n}(x)=\sum_{k=0}^{n}\left(\begin{array}{l}
n \\
k
\end{array}\right) B_{k} x^{n-k} .
$$

The Bernoulli polynomials $B_{n}(x)$ satisfy the difference equation:

$$
B_{n}(x+1)-B_{n}(x)=n x^{n-1} \quad\left(n \in \mathbb{N}_{0}\right),
$$

which yields

$$
B_{n}(0)=B_{n}(1) \quad(n \in \mathbb{N} \backslash\{1\}) .
$$

Setting $x=1$ in (1.3), in view of (1.5), we have

$$
B_{n}=\sum_{k=0}^{n}\left(\begin{array}{l}
n \\
k
\end{array}\right) B_{k}
$$

which gives a recursion formula for computing Bernoulli numbers. The first few of the Bernoulli numbers are listed here:

$$
B_{0}=1, B_{1}=-\frac{1}{2}, B_{2}=\frac{1}{6}, B_{4}=-\frac{1}{30}, B_{6}=\frac{1}{42}, B_{8}=-\frac{1}{30} .
$$

The generalized Bernoulli polynomials $B_{n}^{(\alpha)}(x)$ of degree $n$ in $x$ are defined by the generating function:

$$
\left(\frac{z}{e^{z}-1}\right)^{\alpha} e^{x z}=\sum_{n=0}^{\infty} B_{n}^{(\alpha)}(x) \frac{z^{n}}{n !} \quad\left(|z|<2 \pi ; 1^{\alpha}:=1\right)
$$

for arbitrary (real or complex) parameter $\alpha$. Clearly, we have

$$
B_{n}^{(\alpha)}(x)=(-1)^{n} B_{n}^{(\alpha)}(\alpha-x)
$$

so that

$$
B_{n}^{(\alpha)}(\alpha)=(-1)^{n} B_{n}^{(\alpha)}(0)=:(-1)^{n} B_{n}^{(\alpha)}
$$

in terms of the generalized Bernoulli numbers $B_{n}^{(\alpha)}$ defined by the generating function:

$$
\left(\frac{z}{e^{z}-1}\right)^{\alpha}=\sum_{n=0}^{\infty} B_{n}^{(\alpha)} \frac{z^{n}}{n !} \quad\left(|z|<2 \pi ; 1^{\alpha}:=1\right) .
$$


It is easily observed that

$$
B_{n}^{(1)}(x)=B_{n}(x) \quad \text { and } \quad B_{n}^{(1)}=B_{n} \quad\left(n \in \mathbb{N}_{0}\right) .
$$

We also recall a close relative of the binomial coefficients, the Stirling numbers of the first kind, named after James Stirling (1692-1770). There are also Stirling numbers of the second kind. Although they have a revered and many applications, their notations have not been used in one way, like the binomial coefficient $\left(\begin{array}{l}n \\ k\end{array}\right)$. Following Jovan Karamata [7], Graham et al. [6] have used $\left\{\begin{array}{l}n \\ k\end{array}\right\}$ for Stirling numbers of the second kind and $\left[\begin{array}{l}n \\ k\end{array}\right]$ for Stirling numbers of the first kind by noting that these symbols turn out to be more user-friendly than the many other notations that people have tried. Here we agree to use the notation $\left[\begin{array}{l}n \\ k\end{array}\right]$ for Stirling numbers of the first kind, which counts the number of ways to arrange $n$ objects into $k$ cycles. Note that $\left[\begin{array}{l}n \\ k\end{array}\right]$ is also called the unsigned or absolute Stirling number of the first kind (see, e.g., [2, p. 213]).

This $\left[\begin{array}{l}n \\ k\end{array}\right]$ satisfies the following recurrence:

$$
\left[\begin{array}{l}
n \\
k
\end{array}\right]=(n-1)\left[\begin{array}{c}
n-1 \\
k
\end{array}\right]+\left[\begin{array}{l}
n-1 \\
k-1
\end{array}\right] \quad(n \in \mathbb{N}),
$$

where $\mathbb{N}$ denotes the set of positive integers. We recall some values of $\left[\begin{array}{l}n \\ k\end{array}\right]$ :

$$
\begin{aligned}
& {\left[\begin{array}{l}
n \\
0
\end{array}\right]=[n=0], \quad\left[\begin{array}{l}
n \\
1
\end{array}\right]=(n-1) ! \quad(n \in \mathbb{N}),} \\
& {\left[\begin{array}{l}
n \\
n
\end{array}\right]=1, \quad\left[\begin{array}{c}
n \\
n-1
\end{array}\right]=\left(\begin{array}{l}
n \\
2
\end{array}\right), \quad\left[\begin{array}{l}
n \\
2
\end{array}\right]=(n-1) ! H_{n-1} \quad(n \in \mathbb{N}),}
\end{aligned}
$$

where the notation $[n=m]$ denotes 1 if $n=m$ and 0 otherwise, $H_{n}$ are the harmonic numbers defined by

$$
H_{n}:=\sum_{k=1}^{n} \frac{1}{k} \quad(n \in \mathbb{N}) \quad \text { and } \quad H_{0}:=0 .
$$

For the last identity in (1.14), see also and compare its corresponding identity in [10, p. 77].

Another triangle of values which has often been appeared is due to Leonhard Euler (1707-1783) and called Eulerian numbers denoted by $\left\langle\begin{array}{l}n \\ k\end{array}\right\rangle$. The angle brackets in this case suggests less than and greater than; $\left\langle\begin{array}{c}n \\ k\end{array}\right\rangle$ is the number of permutations $\pi_{1} \pi_{2} \cdots \pi_{n}$ of $\{1,2, \ldots, n\}$ that have $k$ ascents, namely, $k$ places where $\pi_{j}<\pi_{j+1}$. This $\left\langle\begin{array}{l}n \\ k\end{array}\right\rangle$ satisfies the 
following recurrence:

$$
\left\langle\begin{array}{l}
n \\
k
\end{array}\right\rangle=(k+1)\left\langle\begin{array}{c}
n-1 \\
k
\end{array}\right\rangle+(n-k)\left\langle\begin{array}{l}
n-1 \\
k-1
\end{array}\right\rangle \quad(n \in \mathbb{N}) .
$$

We recall some values of $\left\langle\begin{array}{l}n \\ k\end{array}\right\rangle$ :

$$
\begin{aligned}
& \left\langle\begin{array}{l}
n \\
n
\end{array}\right\rangle=[n=0], \quad\left\langle\begin{array}{l}
n \\
k
\end{array}\right\rangle=\left\langle\begin{array}{c}
n \\
n-1-k
\end{array}\right\rangle \quad(n \in \mathbb{N}), \\
& \left\langle\begin{array}{l}
0 \\
k
\end{array}\right\rangle=[k=0] \quad(k \in \mathbb{Z}),
\end{aligned}
$$

where $\mathbb{Z}$ denotes the set of integers.

We recall another triangular pattern of coefficients, shown in Table 270 in [6, p. 270], which are called second-order Eulerian numbers $\left\langle\left\langle\begin{array}{l}n \\ k\end{array}\right\rangle\right\rangle$, because they satisfy a recurrence similar to (1.16) but with $n$ replaced by $2 n-1$ in one place:

$$
\left\langle\left\langle\begin{array}{l}
n \\
k
\end{array}\right\rangle\right\rangle=(k+1)\left\langle\left\langle\begin{array}{c}
n-1 \\
k
\end{array}\right)\right\rangle+(2 n-1-k)\left\langle\left\langle\begin{array}{l}
n-1 \\
k-1
\end{array}\right\rangle\right\rangle \quad(n \in \mathbb{N}) .
$$

Gessel and Stanley [4] first noticed that these second-order Eulerian numbers have a curious combinatorial interpretation (see also $[6, \mathrm{p}$. 270]): If we form permutations of the multiset $\{1,1,2,2, \ldots, n, n\}$ with special property that all numbers between the two occurrences of $m$ are greater than $m$, for $1 \leqslant m \leqslant n$, then $\left\langle\left\langle\begin{array}{l}n \\ k\end{array}\right\rangle\right\rangle$ is the number of such permutations that have $k$ ascents. Graham et al. [6, p. 271] remarked that second-order Eulerian numbers are important chiefly because of their connection with Stirling numbers [5]: For example,

$$
\left[\begin{array}{c}
x \\
x-n
\end{array}\right]=\sum_{k=0}^{n}\left\langle\left\langle\begin{array}{l}
n \\
k
\end{array}\right\rangle\right)\left(\begin{array}{c}
x+k \\
2 n
\end{array}\right) \quad\left(n \in \mathbb{N}_{0}\right),
$$

which holds true whenever $x$ is an integer and $n$ is a nonnegative integer. Since the right-hand side of of (1.19) is a polynomial in $x$, we can use (1.19) to define Stirling numbers of the first kind for arbitrary real (or complex) values of $x$.

If $n \in \mathbb{N}$, the polynomial $\left[\begin{array}{c}x \\ x-n\end{array}\right]$ is zero when $x=0, x=1, \ldots$, and $x=n$; therefore it is divisible by $(x-0),(x-1), \ldots$, and $(x-n)$. Graham et al. [6, p. 271] observed that it is interesting to look at what has left after these known factors are divided out. They define the Stirling polynomials $\sigma_{n}(x)$ by the rule (see [6, p. 271, Eq. (6.45)]):

$$
\sigma_{n}(x)=\left[\begin{array}{c}
x \\
x-n
\end{array}\right] /(x(x-1) \cdots(x-n)) \quad\left(n \in \mathbb{N}_{0}\right),
$$


which is a polynomial in $x$ of degree $n-1$. They [6, p. 272] presented several formulas involving the Stirling polynomials $\sigma_{n}(x)$ which are also defined by the generating function:

$$
\left(\frac{z e^{z}}{e^{z}-1}\right)^{x}=x \sum_{n=0}^{\infty} \sigma_{n}(x) z^{n}
$$

In this paper we aim at presenting certain interesting identities mainly involving the second-order Eulerian numbers and the Stirling polynomials $\sigma_{n}(x)$.

For our purpose, we also recall the following functions. The Psi (or Digamma) function $\psi(z)$ is defined by

$$
\psi(z):=\frac{d}{d z}\{\log \Gamma(z)\}=\frac{\Gamma^{\prime}(z)}{\Gamma(z)} \quad \text { or } \quad \log \Gamma(z)=\int_{1}^{z} \psi(t) d t,
$$

where $\Gamma(z)$ is the familiar Gamma function (see, e.g., $[9$, Section 1.1] and [10, Section 1.1]). For the sake of completeness, we recall the Polygamma functions $\psi^{(n)}(z)(n \in \mathbb{N})$ defined by

$$
\psi^{(n)}(z):=\frac{d^{n+1}}{d z^{n+1}} \log \Gamma(z)=\frac{d^{n}}{d z^{n}} \psi(z) \quad\left(n \in \mathbb{N}_{0} ; z \notin \mathbb{Z}_{0}^{-}\right),
$$

where $\mathbb{Z}_{0}^{-}$denotes the set of nonpositive integers. In terms of the generalized (or Hurwitz) Zeta function $\zeta(s, a)$ (see, e.g., [10, Section 2.2]), we can write

$$
\begin{aligned}
\psi^{(n)}(z) & =(-1)^{n+1} n ! \sum_{k=0}^{\infty} \frac{1}{(k+z)^{n+1}} \\
& =(-1)^{n+1} n ! \zeta(n+1, z) \quad\left(n \in \mathbb{N} ; z \notin \mathbb{Z}_{0}^{-}\right),
\end{aligned}
$$

which may be used to deduce the properties of $\psi^{(n)}(z)(n \in \mathbb{N})$ from those of $\zeta(s, z)(s=n+1 ; n \in \mathbb{N})$ and vice versa.

\section{Identities involving the second-order Eulerian numbers and the Stirling polynomials $\sigma_{n}(x)$}

From (1.19) and (1.20), it is easy to rewrite $\sigma_{n}(x)$ in the following form:

$$
\sigma_{n}(x)=\frac{1}{(n+1) !\left(\begin{array}{c}
x \\
n+1
\end{array}\right)} \sum_{k=0}^{n}\left\langle\left\langle\begin{array}{l}
n \\
k
\end{array}\right\rangle\right\rangle\left(\begin{array}{c}
x+k \\
2 n
\end{array}\right) \quad\left(n \in \mathbb{N}_{0}\right)
$$


By using the expression (see, e.g., $[10$, p. 5]):

$$
\left(\begin{array}{l}
x \\
n
\end{array}\right)=\frac{\Gamma(x+1)}{n ! \Gamma(x-n+1)}
$$

and differentiating each side of (2.1) with respect to the variable $x$, we get

$$
\begin{aligned}
\sigma_{n}^{\prime}(x)= & \frac{1}{(n+1) !\left(\begin{array}{c}
x \\
n+1
\end{array}\right)} \sum_{k=0}^{n}\left\langle\left\langle\begin{array}{l}
n \\
k
\end{array}\right)\right\rangle\left(\begin{array}{c}
x+k \\
2 n
\end{array}\right) \\
& \cdot[(\psi(x-n)-\psi(x+1))+(\psi(x+k+1)-\psi(x+k-2 n+1))] .
\end{aligned}
$$

By using the following known formula for Psi function (see, e.g., [10, p. 25]):

$$
\psi(z+n)=\psi(z)+\sum_{j=1}^{n} \frac{1}{z+j-1} \quad(n \in \mathbb{N}),
$$

a special case of $(2.3)$ when $x=2 n$ yields the following interesting identity:

$$
\begin{aligned}
\sigma_{n}^{\prime}(2 n)= & \frac{(n-1) !}{(2 n) !}\left(H_{n-1}-H_{2 n}\right)\left[\begin{array}{c}
2 n \\
n
\end{array}\right] \\
& +\frac{(n-1) !}{(2 n) !} \sum_{k=0}^{n}\left\langle\left\langle\begin{array}{l}
n \\
k
\end{array}\right\rangle\right\rangle\left(\begin{array}{c}
2 n+k \\
2 n
\end{array}\right)\left(H_{2 n+k}-H_{k}\right),
\end{aligned}
$$

where $H_{n}$ are the harmonic numbers given in (1.15).

The Pochhammer symbol $(\lambda)_{n}$ is defined (for $\lambda \in \mathbb{C}$ ) by (see $[9$, p. 2 and p. 6$]$ and [10, p. 2 and pp. $4-6])$ :

$$
\begin{aligned}
(\lambda)_{n}: & = \begin{cases}1 & (n=0) \\
\lambda(\lambda+1) \ldots(\lambda+n-1) & (n \in \mathbb{N})\end{cases} \\
& =\frac{\Gamma(\lambda+n)}{\Gamma(\lambda)} \quad\left(\lambda \in \mathbb{C} \backslash \mathbb{Z}_{0}^{-}\right),
\end{aligned}
$$

where $\mathbb{C}$ denotes the set of complex numbers. Using (2.6), we have

$$
\left(\begin{array}{l}
\lambda \\
n
\end{array}\right)=\frac{\Gamma(\lambda+1)}{n ! \Gamma(\lambda-n+1)}=\frac{(-1)^{n}(-\lambda)_{n}}{n !} .
$$

Applying (2.7) to (2.1), we obtain $\sigma_{n}(x)$ in the following form:

$$
(-1)^{n+1}(2 n) !(-x)_{n+1} \sigma_{n}(x)=\sum_{k=0}^{n}\left\langle\left\langle\begin{array}{l}
n \\
k
\end{array}\right\rangle\right\rangle(-x-k)_{2 n} \quad\left(n \in \mathbb{N}_{0}\right)
$$


By recalling the following known expansion for $(x)_{n}$ (see [6, p. 264]):

$$
(x)_{n}=\sum_{\ell=0}^{n}\left[\begin{array}{l}
n \\
\ell
\end{array}\right] x^{\ell} \quad\left(n \in \mathbb{N}_{0}\right)
$$

to the right-hand side of (2.8) and using the binomial theorem, we get

$$
\begin{aligned}
(-1)^{n+1}(2 n) !(-x)_{n+1} \sigma_{n}(x) & \\
= & \sum_{k=0}^{n} \sum_{\ell=0}^{2 n} \sum_{j=0}^{\ell}(-1)^{\ell}\left\langle\left\langle\begin{array}{l}
n \\
k
\end{array}\right\rangle\right\rangle\left[\begin{array}{c}
2 n \\
\ell
\end{array}\right]\left(\begin{array}{l}
\ell \\
j
\end{array}\right) k^{\ell-j} x^{j} \\
& =\sum_{k=0}^{n} \sum_{j=0}^{2 n} \sum_{\ell=j}^{2 n}(-1)^{\ell}\left\langle\left\langle\begin{array}{l}
n \\
k
\end{array}\right\rangle\right\rangle\left[\begin{array}{c}
2 n \\
\ell
\end{array}\right]\left(\begin{array}{l}
\ell \\
j
\end{array}\right) k^{\ell-j} x^{j},
\end{aligned}
$$

where, for the second equality, we have used the following rearrangement formula for a double finite series (see, e.g., [1, Eq. (1.24)]):

$$
\sum_{k=0}^{n} \sum_{\ell=0}^{k} A_{k, \ell}=\sum_{\ell=0}^{n} \sum_{k=\ell}^{n} A_{k, \ell} .
$$

Setting $x=r \in \mathbb{N}_{0}$ with $0 \leqslant r \leqslant n-1$ in the right-hand side of (2.10) is easily seen to reduce to zero. So we have the following interesting formulas: For $r \in \mathbb{N}_{0}$ with $0 \leqslant r \leqslant n-1$,

$$
\begin{aligned}
0 & =\sum_{k=0}^{n} \sum_{\ell=0}^{2 n} \sum_{j=0}^{\ell}(-1)^{\ell}\left\langle\left\langle\begin{array}{l}
n \\
k
\end{array}\right)\right\rangle\left[\begin{array}{c}
2 n \\
\ell
\end{array}\right]\left(\begin{array}{l}
\ell \\
j
\end{array}\right) k^{\ell-j} r^{j} \\
& =\sum_{k=0}^{n} \sum_{j=0}^{2 n} \sum_{\ell=j}^{2 n}(-1)^{\ell}\left\langle\left\langle\begin{array}{l}
n \\
k
\end{array}\right\rangle\right\rangle\left[\begin{array}{c}
2 n \\
\ell
\end{array}\right]\left(\begin{array}{l}
\ell \\
j
\end{array}\right) k^{\ell-j} r^{j} .
\end{aligned}
$$

Setting $x=2 n \in \mathbb{N}_{0}$ in the right-hand side of (2.10), we also obtain the following interesting formulas:

$$
\begin{aligned}
\frac{(2 n) !}{n}\left[\begin{array}{c}
2 n \\
n
\end{array}\right] & =\sum_{k=0}^{n} \sum_{\ell=0}^{2 n} \sum_{j=0}^{\ell}(-1)^{\ell+1}\left\langle\left\langle\begin{array}{l}
n \\
k
\end{array}\right\rangle\right\rangle\left[\begin{array}{c}
2 n \\
\ell
\end{array}\right]\left(\begin{array}{l}
\ell \\
j
\end{array}\right) k^{\ell-j}(2 n)^{j} \\
& =\sum_{k=0}^{n} \sum_{j=0}^{2 n} \sum_{\ell=j}^{2 n}(-1)^{\ell+1}\left\langle\left\langle\begin{array}{l}
n \\
k
\end{array}\right\rangle\right\rangle\left[\begin{array}{c}
2 n \\
\ell
\end{array}\right]\left(\begin{array}{l}
\ell \\
j
\end{array}\right) k^{\ell-j}(2 n)^{j} .
\end{aligned}
$$


From (1.21), it is fairly straightforward to deduce the addition theorem:

$$
\sigma_{n}(x+y)=\frac{x y}{x+y} \sum_{k=0}^{n} \sigma_{k}(x) \sigma_{n-k}(y) .
$$

Setting $x=1$ in (1.21) and using (1.1), we have

$$
\sigma_{n}(1)=\frac{B_{n}(1)}{n !} \quad\left(n \in \mathbb{N}_{0}\right)
$$

which, upon considering (1.3) and (1.5), gives

$$
\sigma_{1}(1)=B_{1}(1)=B_{0}+B_{1}=\frac{1}{2} \quad \text { and } \quad \sigma_{n}(1)=\frac{B_{n}}{n !} \quad\left(n \in \mathbb{N}_{0} \backslash\{1\}\right) .
$$

Setting $y=1$ in (2.14) and using (2.16), we have

$$
\sigma_{n}(x+1)=\frac{x}{x+1}\left(\sum_{k=0}^{n-2} \frac{B_{n-k}}{(n-k) !} \sigma_{k}(x)+\frac{1}{2} \sigma_{n-1}(x)+\sigma_{n}(x)\right)
$$

Setting $\alpha=x$ in (1.8) is equal to (1.21). Then equating the coefficients of $z^{n}$ in these two different expressions of the same function, we obtain

$$
\sigma_{n}(x)=\frac{B_{n}^{(x)}(x)}{n ! x} \quad\left(n \in \mathbb{N}_{0}\right) .
$$

Using the relation (2.18) and a known result (see [10, p. 84, Eq.(29)]; see also [11, p. 510, Eq. (3)]), we give the following explicit formula for the Stirling polynomials:

$$
\begin{aligned}
\sigma_{n}(x)= & \frac{1}{n ! x} \sum_{k=0}^{n}\left(\begin{array}{l}
n \\
k
\end{array}\right)\left(\begin{array}{c}
n+k-1 \\
k
\end{array}\right) \frac{k !}{(2 k) !} \sum_{j=0}^{k}(-1)^{j}\left(\begin{array}{l}
k \\
j
\end{array}\right) j^{2 k}(x+j)^{n-k} \\
& \cdot{ }_{2} F_{1}[k-n, k-x ; 2 k+1 ; j /(x+j)],
\end{aligned}
$$

in terms of the Gaussian hypergeometric function ${ }_{2} F_{1}$ (see $[10$, Section $1.5]$; see also [8, Chapter 4]).

\section{Acknowledgements}

The author would like to express his deep gratitude for the reviewer(s) to point out some typographical errors which can be found only through his/her/their detailed reading. 


\section{References}

[1] J. Choi, Notes on formal manipulations of double series, Commun. Korean Math. Soc. 18 (2003), 781-789.

[2] L. Comtet, Advanced Combinatorics: The Art of Finite and Infinite Expansions (Translated from the French by J. W. Nienhuys), Reidel, Dordrecht and Boston, 1974.

[3] J. H. Conway and R. K. Guy, The Book of Numbers, Springer-Verlag, 1996.

[4] I. Gessel and R. P. Stanley, Stirling polynomials, J. Comb. Theor. ser. A 24 (1978), 24-33.

[5] J. Ginsburg, Note on Stirling's numbers, Amer. Math. Monthly 35 (1928), $77-80$.

[6] R. L. Graham, D. E. Knuth, and O. Patashnik, Concrete Mathematics, second edi., Addison-Wesley Publishing Company, 1994.

[7] J. Karamata, Théorèmes sur la sommabilité exponentielle et d'autres sommabilités rattachant, Mathematica (Cluj) 9 (1935), 164-178.

[8] E. D. Rainville, Special Functions, Macmillan Company, New York, 1960; Reprinted by Chelsea Publishing Company, Bronx, New York, 1971.

[9] H. M. Srivastava and J. Choi, Series Associated with the Zeta and Related Functions, Kluwer Academic Publishers, Dordrecht, Boston and London, 2001.

[10] _ Zeta and q-Zeta Functions and Associated Series and Integrals, Elsevier Science Publishers, Amsterdam, London, and New York, 2012.

[11] H. M. Srivastava and P. G. Todorov, An explicit formula for the generalized Bernoulli polynomials, J. Math. Anal. Appl. 130 (1988), 509-513.

[12] J. Worpitzky, Studien über die Beroullischen and Eulerschen Zahlen, J. Reine Angew. Math. 94 (1883), 203-232.

$*$

Department of Mathematics

Dongguk University

Gyeongju 780-714, Republic of Korea

E-mail: junesang@mail.dongguk.ac.kr 